ARTICULO ORIGINAL

\title{
Asociación de las incidencias de hepatoma, las cirrosis y la hepatitis B en Cali
}

\author{
Jorge H. Rojas \\ Secretaría de Salud Pública Municipal de Cali, Colombia.
}

\begin{abstract}
El propósito de la investigación fue medir y comparar las incidencias del hepatoma (carcinoma hepatocelular, $\mathrm{CHC}$ ), la cirrosis hepatocelular y la hepatitis B en la ciudad de Cali y clasificar las comunas de la ciudad según el riesgo de infección y sus complicaciones crónicas, para así establecer prioridades para las comunas más vulnerables y orientar eficientemente las acciones de promoción y prevención. No había estudios similares que expresaran la correlación y dinámica entre estos tres eventos en la región de las Américas.
\end{abstract}

Se revisaron las bases de datos del registro de población de cáncer y de mortalidad de la Secretaría de Salud Pública de Cali correspondientes a 1994; además, la de Vigilancia Epidemiológíca del Programa Ampliado de Inmunizaciones de Cali y del Laboratorio de Salud Pública de Cali de 1996. Se calcularon las incidencias de las enfermedades objeto de estudio según edad, sexo, comuna y Sistema Local de Salud (SILOS) y el coeficiente de correlación de Pearson ( $r$ ) entre ellas, según comunas y silos. Los $r$ fueron altos y significativos estadísticamente según silos, asi: CHC-CIRROSIS, $r=0,82$; IC: 0,03-0,98 y $\mathrm{P}<0,05$; HB-CIRROSIS, $r=0,89$; IC: 0,30,99 y $\mathrm{P}<0,05$; HB-CHC, $r=0,9$; IC: $0,03-0,99$ y $\mathrm{P}<0,05$. Según comunas fue alto y significativo entre $\mathrm{CHC}$ y cirrosis, $\mathrm{r}=0,65$; IC: $0,29-0,85$ y $\mathrm{P}<0,05$; bajo y no significativo estadísticamente entre hepatitis $B$ y las otras dos enfermedades, HB-CIRROSIS, $r=0,22$; IC: $0,02-0,61 ; \mathrm{HB}-\mathrm{CHC}, \mathrm{r}=0,42$; IC: 0,03-0,73 y P>0,05. Estos resultados sugieren una asociación causal entre los eventos. EI cálculo de la costo-efectividad de un programa de inmunización en Nueva York fue 75 veces más favorable que el tratamiento de enfermedades crónicas por hepatitis $B$.

Palabras clave: hepatitis B, hepatoma, cirrosis, correlación.

\section{Association among hepatoma, cirrosis and hepatitis B incidence in Cali}

The purpose of the study was to measure cirrhosis, hepatoma and hepatitis B incidences in Cali, Colombia, and compare them according to geographical area, local health systems (SILOS) and neighborhoods. The coefficients $\mathrm{r}$ were high and statistically significant according to SILOS, as follows: CHC-CIRROSIS, $r=0.82$; IC: $0.03-0.98$ and P<0.05; HB-CIRROSIS, $r=0.89$; IC: $0.3-0.99$ and $\mathrm{P}<0.05$; HB-CHC, $r=0.9$; IC: $0.03-0.99$ and $P<0.05$. With respect to neighborhoods, the coefficient $r$ was high and significant between $\mathrm{CHC}$ and cirrhosis, $r=0.65 ; \mathrm{IC}: 0.29-0.85$ and $P<0.05$, while $r$ were low and not statiscally significant between hepatitis $B$ and the other two diseases: HB-CIRROSIS, $r=0.22$; IC: $0.02-0.61 ; \mathrm{HB}-\mathrm{CHC}, \mathrm{r}=0.42$; IC: $0.03-0.73$ and $\mathrm{P}>0.05$. These results suggest an association among the events described for the three diseases and allow us to priorize the geographic areas for promotion and prevention programs. The cost-effectivity study in the five neighborhoods with the highest risk, based on New York City's surveillance and vaccination program, 1987-1988, showed that immunization is seventy five times better than treatment of liver cronic diseases.

Key words: hepatoma, cirrhosis, hepatitis B, correlation.

Correspondencia: jorgehrojas@telesat.com.co

Recibido: 21/01/00; aceptado: 01/09/00 
El virus de la hepatitis B es el principal agente causal del carcinoma hepatocelular primario $(\mathrm{CHC})$ en todo el mundo (1). Este virus es un carcinógeno reconocido y señalado como el segundo en importancia después del tabaco. Se sabe que causa entre 25 y $67 \%$ de los casos de hepatitis crónica y cirrosis y entre 10 y $70 \%$ de los casos del CHC primario. También es responsable de entre 1 y $16 \%$ de las hepatitis víricas en los niños y entre 25 y $67 \%$ en los adultos (2). Con los estudios longitudinales realizados en Japón y Taiwan, se ha estimado que los portadores del virus de hepatitis $\mathrm{B}$ tienen un riesgo relativo de contraer $\mathrm{CHC}$ primario 200 veces mayor que los no portadores $(1,3)$.

Todavía no se ha determinado la verdadera magnitud de la enfermedad en muchos países de la región de las Américas, ni ha sido posible cuantificar el efecto crónico de la hepatitis $B$ en ninguno de los países, ni fijar las tasas de mortalidad por cirrosis relacionadas con esta infección y con el CHC primario (2). Las tasas de defunción por cirrosis no guardan correlación con las prevalencias del $\mathrm{HBs} A g$ en la región; tampoco se dispone de estudios sobre la cirrosis y el carcinoma hepatocelular en las zonas donde la prevalencia del virus de la hepatitis B es elevada (1).

La hepatitis $B$ es producida por un virus prototipo de la familia hepadnavirus. El virión infeccioso consta de una envoltura de $7 \mathrm{~nm}$ que contiene el antígeno de superficie (AgHbs) y un centro interno de 27 nm (cápside). Esta nucleocápside contiene su propio antígeno core de la hepatitis $\mathrm{B}(\mathrm{AgHBc})$, una molécula de $A D N$ de doble trenza, una polimerasa de ADN endógena y un tercer sistema de antígeno-anticuerpo $(\mathrm{Ag}-\mathrm{Ac})$ relacionado con la infectividad, el Age y AntiHBe de la hepatitis (4).

Los tumores primarios de hígado son relativamente infrecuentes en los Estados Unidos, con una incidencia de $3 / 100.000$ habitantes. Sin embargo, a nivel mundial, estas neoplasias son un serio problema de salud pública. En regiones como Asia y África subsahariana, la incidencia por cada 100.000 habitantes está entre 30 y 150 , y continúa aumentando $(1,2)$ : el pronóstico es grave y la letalidad ocurre en $80 \%$ de los casos (5).

Hay varias evidencias de asociación causal entre el $\mathrm{CHC}$ y la infección por el virus de la hepatitis $\mathrm{B}$.
La variación geográfica muestra una fuerte correspondencia entre las áreas en donde el $\mathrm{CHC}$ es común y el virus de la hepatitis $\mathrm{B}$ es hiperendémico. En China y el surdeste asiático, la prevalencia de la infección por el virus de la hepatitis $B$ es de $100 \%$, la prevalencia de portadores crónicos entre 10 y $15 \%$ y la incidencia del CHC entre 20 y $150 /$ 100.000 habitantes. En Europa y los Estados Unidos, la prevalencia de la infección por el virus está entre 5 y 15\%, la prevalencia de portadores crónicos es menor de $1 \%$ y la incidencia del $\mathrm{CHC}$ entre 1 y 5/100.000 hab. De otro lado, se observa una tasa de prevalencia alta de portadores de $\mathrm{HBsAg}$ entre los enfermos con $\mathrm{CHC}$; entre 85 y $95 \%$ de los pacientes de China y Corea con el $\mathrm{CHC}$ son positivos para el HBsAg. Además, los conglomerados de casos del $\mathrm{CHC}$ en familias con $\mathrm{CHC}$ están relacionados con una alta incidencia del $\mathrm{HBsAg}$ y los miembros afectados usualmente son portadores del mismo. Así mismo, la evidencia más convincente procede de los estudios prospectivos que han demostrado que los portadores del virus de hepatitis $B$ tienen un riesgo más elevado comparados con los controles no infectados, si son apareados por edad y sexo. En Taiwán, ocurrieron 143 casos del $\mathrm{CHC}$ y de cirrosis en 3.454 pacientes con $\mathrm{HBsAg}$ positivo y 9 casos del $\mathrm{CHC}$ y de cirrosis en 19.253 personas con HBsAg negativos. También se ha demostrado la presencia del virus de hepatisis $B$ y otros componentes suyos dentro de los tejidos tumorales examinados (6).

El objetivo de este estudio es realizar una descripción epidemiológica de la hepatitis $B$, las cirrosis y el CHC en Cali, compararlas y determinar la asociación entre ellas. Debido a la escasa información epidemiológica existente sobre la hepatitis B en Cali al inicio del estudio, se decidió promover durante la notificación y clasificación de todos los casos de hepatitis, lo cual permitió recolectar 57 casos nuevos de hepatitis B durante 1996. Se seleccionaron aquellos casos notificados por el laboratorio con HBs Ag positivo (44), con los cuales se hizo el estudio descriptivo, utilizándose la información de archivos de 1994 sobre cirrosis y $\mathrm{CHC}$.

\section{Materiales y métodos}

Se revisó la base de datos del registro de cáncer de la Universidad del Valle en Cali, Colombia, correspondiente a 1994. Se seleccionaron todos 
los casos notificados de cáncer de hígado y vías biliares. Con los docentes del Departamento de Patología de la Universidad del Valle, se estableció la siguiente clasificación: hepatoma $(\mathrm{CHC})$, adenocarcinoma de vías biliares, tumores metastásicos del hígado y hepatoblastoma. Se importó la información para crear una base de datos en Epi Info 5.0. Se seleccionaron todos los casos de residentes en Cali con diagnóstico de $\mathrm{CHC}$ y se elaboró su distribución por comuna, SILOS, sexo y edad. Se calcularon las tasas de incidencia para las variables descritas; se usó como denominador la población mayor de 30 años según las proyecciones de población para Cali en 1994 basadas en el censo de 1973.

Así mismo, se revisó la base de datos de los registros de mortalidad de 1994 de la Secretaría de Salud de Cali, cuya fuente son los certificados de defunción. Se seleccionaron todos los casos de muerte por cirrosis del año 1994 y se estableció la distribución de la mortalidad según comunas, SILOS, sexo y edad. Se calcularon las tasas de mortalidad para las variables descritas; se usó como denominador la población de mayores de 30 años según las proyecciones de población para Cali en 1994 realizadas con base en el censo de 1973.

Igualmente, se revisó la base de datos en Epi Info 5.0 del sistema de vigilancia epidemiológica de la Unidad de Epidemiología de la Secretaría de Salud de Cali. Mediante la ficha epidemiológica, se seleccionaron todos los casos de hepatitis $B$ con resultados positivos de $\mathrm{HBsAg}$, notificados por las instituciones de nivel I, II y III. Además, se adicionaron todos los casos notificados al Laboratorio de Referencia Municipal de Cali por los bancos de sangre de la ciudad. Se calculó la incidencia de la hepatitis B en Cali durante 1996 por comuna, SILOS, sexo y edad; se usó como denominador toda la población de Cali según las proyecciones de población para 1996, realizadas con el censo de 1973.

Se calculó el coeficiente de correlación $r$ de Pearson entre los tres eventos descritos por comuna y SILOS, utilizando Epi Info 5.0, Epi Info 6.04 y MS Excel-Windows. Se utilizó la prueba $t$ de significancia estadística en un nivel de $95 \%$ y se calcularon los intervalos de confianza con la transformación $z$ del valor $r$. Las tasas de incidencia para cirrosis y $\mathrm{CHC}$ se calcularon teniendo como denominador los mayores de 30 años porque se observó que, a partir de esta edad, ocurrieron los casos; para la hepatitis $\mathrm{B}$, se tomó la población de todas la edades porque en los menores de edad también sucedieron casos.

\section{Resultados}

Los promedios de edad de los casos de $\mathrm{CHC}$ y cirrosis fueron similares, 66,3 años (IC: 62,6 a 69,9) y 63,9 años $(61,6-66,4)$, respectivamente; las medianas de edad de los casos de $\mathrm{CHC}$ y cirrosis fueron semejantes, 68 y 67,5 años, respectivamente. Las desviaciones estándares para los tres eventos también fueron similares, 14,9, 13,4 y 14,7 , respectivamente. El promedio de la edad para los casos de hepatitis B fue 32,9 años y la mediana 29 años.

La distribución geográfica por SILOS de la incidencia del carcinoma hepatocelular primario (CHC) mostró que el SILOS 3 tuvo mayor riesgo con una incidencia entre 8,5 y 10 casos por 100.000 habitantes mayores de 30 años; los SILOS 1, 2, 4 y 5 se ubicaron en un riesgo intermedio con incidencias entre 5,0 y 8,4 casos por 100.000 habitantes mayores de 30 años, y el SILOS 6 en riesgo más bajo con incidencias entre 0,0 y 4,9 casos por 100 mil hab mayores de 30 años (cuadro 1).

Cuadro 1. Tasas de mortalidad por hepatoma y cirrosis en 1994 y de incidencia de la hepatitis B en 1996 por SILOS de Cali.

\begin{tabular}{cccc}
\hline SILOS & Hepatoma* $^{*}$ & Cirrosis $^{*}$ & Hepatitis B $^{* *}$ \\
\hline 1 & 6,3 & 15,3 & 2,2 \\
2 & 10,0 & 20,7 & 2,2 \\
3 & 18,0 & 32,1 & 5,1 \\
4 & 9,5 & 24,2 & 2,8 \\
5 & 7,8 & 4,9 & 1,1 \\
6 & 6,6 & 9,3 & 2,2 \\
\hline
\end{tabular}

* Tasa/100.000 hab mayores de 30 años

** Tasa/100.000 hab de todas las edades

Fuente: Programa de Vigilancia Epidemiológica de Enfermedades Inmunoprevenibles, Unidad de Epidemiología y Salud Pública, Secretaría de Salud Pública Municipal de Cali. 
La distribución geográfica por SILOS de la mortalidad por cirrosis mostró que el SILOS 3 tuvo mayor riesgo con una incidencia entre 25,1 y 32,1 casos por 100.000 personas mayores de 30 años; Ios SILOS 1, 2 y 4, un riesgo intermedio con incidencias entre 10,6 y 25 por 100.000 habitantes mayores de 30 años, y los SILOS 5 y 6 , riesgo más bajo con incidencias entre 0,0 y 10,4 casos por 100.000 personas mayores de 30 años.

La distribución geográfica por SILOS de la incidencia de la hepatitis B mostró que el SILOS 3 tuvo mayor riesgo con una incidencia entre 3,5 y 5,1 casos por 100.000 hab; los SILOS 1, 2, 4 y 6 un riesgo intermedio con incidencias entre 1,4 y 3,5 casos por 100.000 hab, y el SILOS 5 riesgo más bajo con incidencias entre 0,0 y 1,4 casos por 100.000 hab.

La distribución geográfica por comunas también permitió clasificarlas según el riesgo (cuadros 2 y 3$)$.

Cuadro 2. Tasas de mortalidad por hepatoma y cirrosis en 1994 y de incidencia de hepatitis B en 1996 por comunas de Cali.

\begin{tabular}{cccc}
\hline Comuna & Hepatoma* $^{*}$ & Cirrosis $^{*}$ & Hepatitis $\mathbf{B}^{\text {** }}$ \\
\hline 1 & 0,0 & 9,9 & 0,0 \\
2 & 2,1 & 21,0 & 1,8 \\
3 & 3,5 & 21,0 & 1,6 \\
4 & 23,0 & 27,0 & 1,4 \\
5 & 8,4 & 25,0 & 7,5 \\
6 & 8,1 & 16,0 & 0,8 \\
7 & 12,0 & 15,4 & 0,0 \\
8 & 18,0 & 25,6 & 5,9 \\
9 & 20,0 & 43,6 & 7,3 \\
10 & 11,0 & 33,0 & 3,0 \\
11 & 10,0 & 21,0 & 4,7 \\
12 & 19,0 & 28,1 & 1,6 \\
13 & 5,0 & 7,4 & 2,2 \\
14 & 14,0 & 2,7 & 0,0 \\
15 & 4,0 & 4,0 & 0,9 \\
16 & 10,0 & 16,8 & 1,0 \\
17 & 5,5 & 11,0 & 0,0 \\
18 & 4,9 & 4,9 & 8,0 \\
19 & 11,0 & 18,7 & 3,3 \\
20 & 0,0 & 0,0 & 1,6 \\
\hline
\end{tabular}

* Tasa/100.000 hab mayores de 30 años

** Tasa/100.000 hab de todas las edades

Fuente: Programa de Vigilancia Epidemiológica de Enfermedades Inmunoprevenibles, Unidad de Epidemiologia y Salud Pública, Secretaría de Salud Pública Municipal de Cali.
Cuadro 3. Muertes por hepatoma y cirrosis en 1994 y casos de hepatitis B en 1996 por comunas de Cali.

\begin{tabular}{cccc}
\hline Comuna & $\begin{array}{c}\text { Hepatoma } \\
\mathbf{n}\end{array}$ & $\begin{array}{c}\text { Cirrosis } \\
\mathbf{n}\end{array}$ & $\begin{array}{c}\text { Hepatitis B } \\
\mathbf{n}\end{array}$ \\
\hline 1 & 0 & 1 & 0 \\
2 & 1 & 10 & 2 \\
3 & 1 & 6 & 1 \\
4 & 6 & 7 & 1 \\
5 & 2 & 6 & 5 \\
6 & 3 & 6 & 1 \\
7 & 3 & 4 & 0 \\
8 & 7 & 10 & 6 \\
9 & 6 & 13 & 5 \\
10 & 4 & 12 & 3 \\
11 & 3 & 6 & 4 \\
12 & 4 & 6 & 1 \\
13 & 2 & 3 & 3 \\
14 & 5 & 1 & 0 \\
15 & 1 & 1 & 1 \\
16 & 3 & 5 & 1 \\
17 & 3 & 6 & 0 \\
18 & 1 & 1 & 5 \\
19 & 6 & 10 & 4 \\
20 & 0 & 0 & 1 \\
\hline
\end{tabular}

Fuente: Programa de Vigilancia de Enfermedades Inmunoprevenibles, Unidad de Epidemiología y Salud Pública, Secretaría de Salud Pública Municipal de Cali.

Se calculó el coeficiente r de Pearson y se hallaron valores muy altos de correlación por SILOS entre la incidencia de $\mathrm{CHC}$ y la mortalidad por cirrosis $(r=0,82$; IC: 0,03 a 0,$98 ; p<0,05)$; entre la mortalidad por cirrosis y la incidencia de hepatitis $B(r=0,89$; IC: 0,03 a 0,$99 ; p<0,05)$, y entre la incidencia de hepatoma y la incidencia de hepatitis $B$ $(r=0,90$; IC: 0,03 a 0,$99 ; p<0,05)$.

La correlación por comunas entre la incidencia de hepatoma y la mortalidad por cirrosis fue alta $(r=0,65$; IC: 0,29 a 0,$85 ; p<0,05)$. Hubo correlaciones intermedias entre la mortalidad por cirrosis y la incidencia de hepatitis B ( $r=0,22 ; \mathrm{IC}: 0,02$ a $0,61 ; p>0,05)$, y entre la incidencia de hepatoma y la incidencia de hepatitis B ( $r=0,42 ; I C: 0,03$ a 0,$73 ; p>0,05$ ).

\section{Discusión}

El estudio realizado es de tipo ecológico, es decir, las unidades de análisis son comunidades y no individuos; por tanto, se debe tener precaución al interpretar estos resultados para no hacer inferencias a nivel individual, cayendo en la falacia 
ecológica. La distribución de los eventos según la edad evidencia que la hepatitis B antecede a la cirrosis y al $\mathrm{CHC}$ entre 30 y 35 años y que la cirrosis precede al $\mathrm{CHC}$ entre 2 y 3 años, lo cual corrobora la relación tiempo-respuesta descrito en la literatura. La incidencia de los eventos en Cali según comuna y SILOS evidencia la relación dosis-respuesta y está de acuerdo con lo ocurrido en otras latitudes según la literatura científica (2); los hallazgos de la distribución por grupos de edad y la focalización en áreas geográficas con mayor riesgo de transmisibilidad de la enfermedad y de endemicidad, como ocurrió en las comunas 4, 8 , 9,12 y 7 , permiten establecer las prioridades para las acciones de promoción y protección.

La ausencia de significancia estadística entre los coeficientes de correlación $r$ de Pearson, calculados por comunas para asociar el $\mathrm{CHC}$ y la hepatitis $B$, y la cirrosis y la hepatitis $B$, se explica porque se comparan dos eventos que son la expresión de procesos en estadios diferentes, uno crónico y el otro agudo o en las fases iniciales de la cronicidad que ocurrieron con 30 años de intervalo el uno del otro, por lo que la interpretación debe ser cuidadosa. Los hallazgos sugieren que cuando la punta del iceberg surge (hepatomas y cirrosis clínicas), ya han transcurrido cerca de 30 años $(9,10)$, durante los cuales la enfermedad se ha expandido a otras comunas. Esta observación puede respaldarse con la distribución de los casos de hepatitis B en las comunas 5 y 18 en 1996, la cual no muestra incidencias altas de $\mathrm{CHC}$ y cirrosis y también por la ocurrencia de importaciones causadas por fenómenos migratorios que disminuyen la fuerza de la correlación. El hallazgo de correlación por SILOS se debe probablemente a que éstos, por corresponder a espacios geográficos más grandes que las comunas, no se ven tan afectados por las migraciones. Contar con un número mayor de localidades para correlacionar también podría aclarar la significancia.

Durante 1994 se registraron 10.437 muertes en Cali, de las cuales 1.424 ocurrieron por cáncer, 106 por cirrosis y 61 por $\mathrm{CHC}(12,13)$. Si se asume que la supervivencia de un caso de $\mathrm{CHC}$ es entre 3 y 6 meses, la suma de las muertes por $\mathrm{CHC}$ y cirrosis en 1994 en Cali fue de 167 casos. Si entre
12 y $70 \%$ de los casos de $\mathrm{CHC}$ y entre 15 y $63 \%$ de las muertes por cirrosis son causadas por la infección del virus de la hepatitis B (3), entonces, 110 de estas muertes estuvieron asociadas con el virus $\mathrm{HB}$, las cuales se originaron en 25 a $30 \%$ de los portadores crónicos del virus de la hepatitis B (8) existentes en 1974, estimados en 367.

En muchas regiones del mundo, como Italia y Japón, otros factores y los virus no-A no-B probablemente juegan un papel mayor en la etiología del CHC (11).

Una revisión de 8.760 codificaciones realizadas y archivadas desde enero de 1953 hasta junio de 1984 en el Servicio de Patología del Hospital Universitario del Valle (HUV), informó 59 casos de tumores primarios de hígado. Dos terceras partes $(67 \%)$ de los casos de hepatoma de adulto estaban asociados con cirrosis y sólo en 2 casos la cirrosis fue clasificada como posthepatítica o postnecrótica. Esta información concuerda con observaciones no publicadas que informan una proporción de $50 \%$ de positividad en la coloración de orceína, mediante el método de Shikata, en los hepatomas examinados, lo cual sugiere que la mayoría de los casos de hepatoma de Cali estaban asociados etiológicamente con el virus de la hepatitis $B(14)$.

Los costos médicos para un paciente con enfermedad hepática crónica (cirrosis), sin trasplante, son de U.S.\$87.000 $(15,16)$. Basados en un estudio llevado a cabo en la ciudad de Nueva York de 1987 a 1988, y aplicando la prevalencia de HBsAg $(2,4 \%)$ hallada en dicho estudio al tamizaje realizado a 20.108 mujeres embarazadas de Cali (17), el número estimado de eventos prevenibles por vacunación en las cinco comunas de mayor riesgo suma 91 (cuadro 4). El costo total de los tratamientos, sin incluir el trasplante, sería de U.S. $\$ 7^{\prime} 917.000$. Si el costo de 3 dosis de vacuna de hepatitis $B$ recombinante es de U.S.\$2,46 (18), se requerirían U.S.\$104.788 para vacunar 42.597 recién nacidos cada año. Finalmente, hay una razón de costo-efectividad 75 veces más favorable para un programa preventivo de vacunación. Por consiguiente, se sugiere: 1) promover la notificación y clasificación de todos los casos de hepatitis; 2) estudiar en las comunas los grupos de población de mayor riesgo 
Cuadro 4. Eventos crónicos prevenibles por inmunización contra hepatitis $B$ en las cinco comunas de mayor riesgo de Cali, 2000.

\begin{tabular}{rccc}
\hline Comuna & $\begin{array}{c}\text { Nacidos } \\
\text { vivos }\end{array}$ & $\begin{array}{c}\text { Portadores de } \\
\text { HbsAg }\end{array}$ & $\begin{array}{c}\text { Eventos } \\
\text { crónicos } \\
\text { prevenibles } \\
\mathbf{n}\end{array}$ \\
\hline 8 & 2.918 & 25 & 23 \\
9 & 1.653 & 14 & 13 \\
12 & 2.209 & 19 & 18 \\
7 & 2.738 & 24 & 22 \\
4 & 1.836 & 16 & 15 \\
Total & 11.354 & 98 & 91 \\
\hline
\end{tabular}

Fuente: Proyecciones de población estadística, Secretaría de Salud de Cali. Am J Public Health 1992;82(6).

para establecer criterios de prioridades, mecanismos y fuentes de transmisión que orienten las acciones de promoción, prevención y control; 3) mejorar las condiciones de vigilancia de la tasa de circulación viral en las comunidades, y 4) conducir estudios más profundos sobre la asociación entre las hepatitis, el $\mathrm{CHC}$ y la cirrosis.

\section{Agradecimientos}

El autor agradece a los profesores de la Facultad de Salud de la Universidad del Valle que de alguna manera contribuyeron a la elaboración de este documento y al personal de la Secretaría de Salud Pública Municipal de Cali por su colaboración.

\section{Referencias}

1. Comisión Ad Hoc sobre la hepatitis vírica. Hepatitis vírica en la región. Bol Oficina Sanit Panam 1986;100:330-7.

2. Hadler S, Fay O, Pinhero F, Maynard J. La hepatitis en las Américas: Informe del Grupo Colaborador de la OPS. Bol Oficina Sanit Panam 1987;103:185-209.

3. Mayer R. Cáncer del aparato digestivo. Medicina Sci Amer $1988 ; 5: 2442-4$

4. Blaine F. Hepatitis B virus. En: Fields N, editor. Virology. 2nd. edition. New York; 1990.
5. Niederhuber J. Tumors of the liver. En: Murphy G, Lawrence W Jr, Lenhard R Jr, editors. Textbook of Clinical Oncology. 2nd. edition. Atlanta, Georgia: American Cancer Society; 1995. p.269-80.

6. Di Biscegli A, Rustgi V, Hoofnagle J. Hepatocelular carcinoma. Ann Intern Med 1988;108:390-401.

7. Laboratorio de Referencia de la Secretaría Municipal de Salud de Cali. Estadística de la red de bancos de sangre de Cali. Cali; 1995.

8. Benenson A. Carcinoma hepatocelular. En: Manual para el control de las enfermedades transmisibles. $16^{\mathrm{a}}$ ed. Washington: OPS; 1997. p.325-7.

9. Castrillón J, Arroyave A. Hepatitis B: una revisión sobre el tema. Bol Epidemiol Antioquia 1994;19:342-63.

10. Universidad del Valle, Facultad de Salud. Registro de Población de Cáncer de Cali. Cali,1994.

11. Oficina de información y estadística de la Secretaría Municipal de Salud de Cali. Mortalidad de Cali, 1994 Secretaría de Salud de Cali, 1994.

12. Hoyos A, Ramírez V, González A. Hepatitis B: inmunogenecidad de la vacuna recombinante cubana anti HBV en trabajadores de la salud vacunados sin seroprotección. Biomédica 1991;11:61-4.

13. Simmonetti R, Camma C, Fiorello F. Hepatitis C virus infection as a risk factor for hepatocelular carcinoma in patients with cirrhosis. Ann Intern Med 1992;116:97-102.

14. Moreno C, De Lima E, Villarraga H, Cuello C, Mayoral L. Hepatoma: experiencia en Cali. Colombia Médica 1986;17:9-15.

15. Margolis H, Coleman P, Brown R. Prevention of hepatitis $B$ virus transmission by immunization. JAMA 1995;274 1201-8.

16. CDC. Prevention of perinatal hepatitis $B$ through enhanced case managment-Connecticut, 1994-1995, and United States, 1994. MMWR 1996;45:585-7.

17. Henning $\mathbf{K}$, Pollak D, Friedman S. A neonatal hepatitis $B$ surveillance and vaccination program: New York City, 1987 to 1988. Am J Public Health 1992;82:885-8.

18. Programa Ampliado de Inmunizaciones. Precios de 1999 del Fondo Rotatorio para la Compra de Vacunas. Boletín informativo PAI/OPS 1998;20:8. 